\title{
On CLUR points of Orlicz spaces
}

\author{
by QUANDI WANG (Changchun), \\ Liang Zhao (Harbin) and Tingfu Wang (Harbin)
}

\begin{abstract}
Criteria for compactly locally uniformly rotund points in Orlicz spaces are given.
\end{abstract}

Let $[X,\|\cdot\|]$ be a Banach space. $S(X)$ and $B(X)$ are the unit sphere and unit ball of $X$ respectively. A point $x$ on $S(X)$ is said to be a locally uniformly rotund point (LUR point) provided that $\left\{x_{n}\right\} \subset X,\left\|x_{n}\right\| \rightarrow 1$ and $\left\|x_{n}+x\right\| \rightarrow 2$ imply $\left\|x_{n}-x\right\| \rightarrow 0$. Recently Y. A. Cui, H. Hudzik and C. Meng [2] introduced the concept of compactly locally uniformly rotund point (CLUR point for short). $x \in S(X)$ is said to be a CLUR point provided that $\left\{x_{n}\right\} \subset X,\left\|x_{n}\right\| \rightarrow 1$ and $\left\|x_{n}+x\right\| \rightarrow 2$ imply that $\left\{x_{n}\right\}$ is a compact set. Obviously, if every point on $S(X)$ is LUR (CLUR) then $X$ is a LUR (CLUR) space. In 1975, B. B. Panda and O. P. Kapoor [4] proved that CLUR implies the Kadec-Klee property and $X$ is LUR iff $X$ is CLUR and strictly rotund. In 1984, J. Y. Fu and W. Y. Zhang [3] showed that if $X^{* *}$ is CLUR and $K$ is a non-null closed, convex Chebyshev set in $X$ then the projection on $K$ is continuous. Y. A. Cui et al. [2] obtained a criterion for a point to be CLUR in Orlicz sequence spaces equipped with the Luxemburg norm.

In this paper we will discuss the CLUR points in Orlicz sequence spaces equipped with the Orlicz norm and in Orlicz function spaces equipped with both the Luxemburg and Orlicz norm.

A mapping $M:(-\infty, \infty) \rightarrow[0, \infty)$ is said to be an $N$-function if it is even, convex, vanishing only at zero and such that $\lim _{u \rightarrow 0} M(u) / u=0$ and $\lim _{u \rightarrow \infty} M(u) / u=\infty$. An interval $[a, b]$ is called a structurally affine interval (SAI for short) of $M$ provided that $M$ is affine on $[a, b]$ and it is not affine on $[a-s, b]$ or $[a, b+s]$ for any $s>0$. For an $N$-function $M$ we

2000 Mathematics Subject Classification: 46B20, 46E30.

Key words and phrases: Orlicz spaces, compactly locally uniformly rotund. 
define its complementary function by

$$
N(v)=\sup _{u>0}\{u|v|-M(u)\} \quad(v \in(-\infty, \infty)) .
$$

This is again an $N$-function (see [1]). Denote by $p(u), p_{-}(u)$ the right and left derivative of $M(u)$ respectively. We have [1] the so-called Young inequality

$$
u v \leq M(u)+N(v)
$$

for any $u, v \geq 0$, and $u v=M(u)+N(v)$ iff $p_{-}(u) \leq v \leq p(u)$.

$M$ is said to satisfy the $\Delta_{2}$-condition ( $M \in \Delta_{2}$, for short) provided there are $k>2$ and $u_{0}>0$ such that $M(2 u) \leq k M(u)$ for $u$ satisfying $u \geq u_{0}$. $M \in \nabla_{2}$ means that $N \in \Delta_{2}$.

Let $(T, \Sigma, \mu)$ be a nonatomic finite measure space and $L^{0}$ the set of all $\Sigma$-measurable functions $x: T \rightarrow(-\infty, \infty)$. For a given $N$-function $M$, the modular of $x$ with respect to $M$ is $\varrho_{M}(x)=\int_{T} M(x(t)) d \mu$. The linear set

$$
\left\{x \in L^{0}: \exists \lambda>0 \text { such that } \varrho_{M}(\lambda x)<\infty\right\}
$$

equipped with the Luxemburg norm

$$
\|x\|_{M}=\inf \left\{\lambda>0: \varrho_{M}(x / \lambda) \leq 1\right\}
$$

or Orlicz norm

$$
\|x\|_{M}^{0}=\sup \left\{\int_{T} x(t) y(t) d \mu: \varrho_{N}(y) \leq 1\right\}=\inf _{k>0} \frac{1}{k}\left(1+\varrho_{M}(k x)\right)
$$

is a Banach space and is called an Orlicz space denoted by $L_{M}$ or $L_{M}^{0}$ respectively.

For $0 \neq x \in L_{M}^{0}$, define

$$
\begin{aligned}
k_{x}^{*} & =\inf \left\{k>0: \varrho_{N}(p(k|x|))=\int_{T} N(p(k|x(t)|)) d \mu \geq 1\right\}, \\
k_{x}^{* *} & =\sup \left\{k>0: \varrho_{N}(p(k|x|)) \leq 1\right\} .
\end{aligned}
$$

It is known that $\|x\|_{M}^{0}=k^{-1}\left(1+\varrho_{M}(k x)\right)$ iff $k \in\left[k_{x}^{*}, k_{x}^{* *}\right]$ (see [1], Th. 1.31).

Similarly we denote by $I_{M}$ and $I_{M}^{0}$ the Orlicz sequence spaces equipped with the Luxemburg norm and Orlicz norm respectively. We only need to notice that $M \in \Delta_{2}$ in the sequence case means that there exist $k>2$ and $u_{0}>0$ such that $M(2 u) \leq k M(u)$ for all $u$ satisfying $|u| \leq u_{0}$.

The following is known ([2], Th. 6): $x \in S\left(I_{M}\right)$ is a CLUR point iff (a) $M \in \Delta_{2}$, (b) $M \in \nabla_{2}$ or $\{i:|x(i)| \in(a, b]\}=\emptyset$ for any SAI $[a, b]$ of $M$.

A criterion for CLUR points in $I_{M}^{0}$ is different.

Theorem 1. Let $x \in S\left(I_{M}^{0}\right)$. Then $x$ is a CLUR point iff (a) $M \in \Delta_{2}$, (b) $M \in \nabla_{2}$. 
Pr o of. Necessity. Suppose that $1=\|x\|_{M}^{0}=k^{-1}\left(1+\varrho_{M}(k x)\right)$. Assume $M \notin \Delta_{2}$. If $\lim _{n \rightarrow \infty}\left\|x-[x]_{n}\right\|_{M}^{0}=0$, we take

$$
z \in B\left(I_{M}\right), \quad \lim _{n \rightarrow \infty}\left\|\frac{z-[z]_{n}}{k}\right\|_{M}^{0}>0
$$

if $\lim _{n \rightarrow \infty}\left\|x-[x]_{n}\right\|_{M}^{0}>0$, we take $z=0$. Then we always have

$$
\lim _{n \rightarrow \infty}\left\|\left(x-\frac{z}{k}\right)-\left[x-\frac{z}{k}\right]_{n}\right\|_{M}^{0}>0,
$$

where $[x]_{n}=(x(1), \ldots, x(n), 0,0, \ldots)$. Let

$$
x_{n}=\left(x(1), \ldots, x(n), \frac{z(n+1)}{k}, \frac{z(n+2)}{k}, \ldots\right) \quad(n=1,2, \ldots) .
$$

Then

$$
\begin{aligned}
\left\|x_{n}\right\|_{M}^{0} & \leq \frac{1}{k}\left(1+\varrho_{M}\left(k x_{n}\right)\right) \\
& =\frac{1}{k}\left(1+\sum_{i=1}^{n} M(k x(i))+\sum_{i>n} M(z(i))\right) \\
& \rightarrow \frac{1}{k}\left(1+\varrho_{M}(k x)\right)=1
\end{aligned}
$$

and

$$
\left\|x_{n}+x\right\|_{M}^{0} \geq 2\left\|[x]_{n}\right\|_{M}^{0} \rightarrow 2\|x\|_{M}^{0}=2 .
$$

Clearly if $\left\{x_{n}\right\}$ has a convergent subsequence $\left\{x_{n_{j}}\right\}$, then $\left(x_{n_{j}}\right)$ has to converge to $x$. But

$$
\lim _{j \rightarrow \infty}\left\|x-x_{n_{j}}\right\|_{M}^{0}=\lim _{j \rightarrow \infty}\left\|\left(x-\frac{z}{k}\right)-\left[x-\frac{z}{k}\right]_{n_{j}}\right\|_{M}^{0}>0
$$

which is a contradiction. Thus (a) is true.

If $M \notin \nabla_{2}$, then there exist

$$
v_{n}<N^{-1}\left(\frac{1}{n+1}\right), \quad N\left(\left(1+\frac{1}{n}\right) v_{n}\right)>(n+1) N\left(v_{n}\right) \quad(n=1,2, \ldots) .
$$

Take the positive integer $m_{n}$ satisfying

Let

$$
\frac{1}{n+1}<m_{n} N\left(v_{n}\right) \leq \frac{1}{n} \quad(n=1,2, \ldots) .
$$

$$
y_{n}=(\overbrace{0, \ldots, 0}^{m_{1}+\ldots+m_{n-1}}, \overbrace{v_{n}, \ldots, v_{n}}^{m_{n}}, 0,0, \ldots) \quad(n=1,2, \ldots) .
$$

Then $\operatorname{supp} y_{n} \cap \operatorname{supp} y_{j}=\emptyset(n \neq j)$. Since $\varrho_{N}\left(y_{n}\right)=m_{n} N\left(v_{n}\right)<1$ and

$$
\varrho_{N}\left((1+1 / n) y_{n}\right)=m_{n} N\left((1+1 / n) v_{n}\right)>(n+1) m_{n} N\left(v_{n}\right)>1,
$$


we have

$$
1 \geq\left\|y_{n}\right\|_{N} \geq \frac{1}{1+1 / n} \quad(n=1,2, \ldots) .
$$

From [1], Prop. $1.83, y_{n}$ has a supporting function $x_{n}$ on $S\left(I_{M}^{0}\right)$, i.e. $\left\|x_{n}\right\|_{M}^{0}$ $=1$,

$$
\left\langle x_{n}, y_{n}\right\rangle=\sum_{i} x_{n}(i) y_{n}(i)=\left\|y_{n}\right\|_{N}>\frac{1}{1+1 / n}
$$

and $\operatorname{supp} x_{n}=\operatorname{supp} y_{n}(n=1,2, \ldots)$.

From [1], Th. 1.45, there is $y \in S\left(L_{N}\right)$ with $\langle x, y\rangle=\|x\|_{M}^{0}=1$. Let

$$
z_{n}=\frac{1}{1+1 / n}(y(1), \ldots, y\left(\sum_{k=1}^{n-1} m_{k}\right), \overbrace{v_{n}, \ldots, v_{n}}^{m_{n}}, y\left(\sum_{k=1}^{n} m_{k}+1\right), \ldots) .
$$

Then

$$
\begin{aligned}
\varrho_{N}\left(z_{n}\right) & \leq \frac{1}{1+1 / n}\left(\varrho_{N}(y)+m_{n} N\left(v_{n}\right)\right) \\
& \leq \frac{1}{1+1 / n}(1+1 / n)=1 \quad(n=1,2, \ldots) .
\end{aligned}
$$

Hence

$$
\begin{aligned}
\left\|x+x_{n}\right\|_{M}^{0} \geq & \left\langle x+x_{n}, z_{n}\right\rangle \\
= & \frac{1}{1+1 / n}\left(\sum_{i=1}^{\infty} x(i) y(i)\right. \\
& \left.-\sum_{i=m_{1}+\ldots+m_{n-1}+1}^{m_{1}+\ldots+m_{n}}\left(x(i) y(i)-x(i) v_{n}-x_{n}(i) y_{n}(i)\right)\right) \\
\geq & \frac{1}{1+1 / n}\left(\langle x, y\rangle+\left\langle x_{n}, y_{n}\right\rangle\right. \\
& \left.-\sum_{i=m_{1}+\ldots+m_{n-1}+1}^{m_{1}+\ldots+m_{n}}\left(|x(i) y(i)|+|x(i)| v_{n}\right)\right) \\
\geq & \frac{1}{1+1 / n}\left(1+\frac{1}{1+1 / n}\right. \\
& \left.-\sum_{i=m_{1}+\ldots+m_{n-1}+1}^{m_{1}+\ldots+m_{n}}(2 M(x(i))+N(y(i)))-m_{n} N\left(v_{n}\right)\right) \\
\rightarrow & 1 \quad(n \rightarrow \infty) .
\end{aligned}
$$

But since $\operatorname{supp} x_{n} \cap \operatorname{supp} x_{j}=\emptyset(n \neq j)$, we have $\left\|x_{n}-x_{j}\right\|_{M}^{0} \geq\left\|x_{n}\right\|_{M}^{0}=1$.

This means $\left\{x_{n}\right\}$ is not a compact set. Thus (b) is true. 
Sufficiency. Assume

$1=\|x\|_{M}^{0}=\frac{1}{k}\left(1+\varrho_{M}(k x)\right)=\left\|x_{n}\right\|_{M}^{0}=\frac{1}{k_{n}}\left(1+\varrho_{M}\left(k_{n} x_{n}\right)\right) \quad(n=1,2, \ldots)$

and $\left\|x_{n}+x\right\|_{M}^{0} \rightarrow 2$. Firstly we will prove that

$$
\lim _{j_{0} \rightarrow \infty} \sup _{n} \sum_{j>j_{0}} M\left(k_{n} x_{n}(j)\right)=0 .
$$

Otherwise there exist $j_{n} \rightarrow \infty$ and $\varepsilon>0$ such that $\sum_{j>j_{n}} M\left(k_{n} x_{n}(j)\right) \geq \varepsilon$ $(n=1,2, \ldots)$. From $M \in \nabla_{2}$ and [1], Th. 1.35, $\bar{k}=\max \left\{k, \sup _{n} k_{n}\right\}<\infty$. Again from $M \in \nabla_{2}$ and [1], Th. 1.13, there exists $\delta \in(0,1)$ such that

$$
M(\lambda u) \leq(1-\delta) \lambda M(u) \quad\left(\lambda \leq \frac{\bar{k}}{1+\bar{k}},|u| \leq \bar{k} M^{-1}(1)\right) .
$$

From $M \in \Delta_{2}$ and [1], Th. 1.40, there exists $\eta>0$ such that for $\varrho_{M}(x) \leq \bar{k}$ and $\varrho_{M}(y) \leq \eta$,

$$
\left|\varrho_{M}(x+y)-\varrho_{M}(x)\right| \leq \frac{\delta \varepsilon}{2(1+\bar{k})} .
$$

Since $k k_{n} /\left(k+k_{n}\right) \leq \bar{k} / 2$ and $M \in \Delta_{2}$,

$$
\sum_{j>j_{n}} M\left(\frac{k k_{n}}{k+k_{n}} x(j)\right)<\sum_{j>j_{n}} M\left(\frac{\bar{k}}{2} x(j)\right)<\eta
$$

for $n$ large enough. Hence for $n$ large enough, by (2)-(4),

$$
\begin{aligned}
0 \leftarrow & \left\|x_{n}\right\|_{M}^{0}+\|x\|_{M}^{0}-\left\|x+x_{n}\right\|_{M}^{0} \\
\geq & \frac{1}{k_{n}}\left(1+\varrho_{M}\left(k_{n} x_{n}\right)\right)+\frac{1}{k}\left(1+\varrho_{M}(k x)\right) \\
& -\frac{k+k_{n}}{k k_{n}}\left(1+\varrho_{M}\left(\frac{k k_{n}}{k+k_{n}}\left(x+x_{n}\right)\right)\right) \\
= & \frac{k+k_{n}}{k k_{n}} \sum_{j=1}^{\infty}\left(\frac{k}{k+k_{n}} M\left(k_{n} x_{n}(j)\right)+\frac{k}{k+k_{n}} M(k x(j))\right. \\
& \left.-M\left(\frac{k k_{n}}{k+k_{n}}\left(x(j)+x_{n}(j)\right)\right)\right) \\
\geq & \frac{k+k_{n}}{k k_{n}}\left(\sum_{j>j_{n}}\left(\frac{k}{k+k_{n}} M\left(k_{n} x_{n}(j)\right)+\frac{k}{k+k_{n}} M(k x(j))\right)\right. \\
& \left.-\sum_{j>j_{n}} M\left(\frac{k k_{n}}{k+k_{n}}\left(x(j)+x_{n}(j)\right)\right)\right)
\end{aligned}
$$




$$
\begin{aligned}
\geq & \frac{k+k_{n}}{k k_{n}}\left(\sum_{j>j_{n}} \frac{k}{k+k_{n}} M\left(k_{n} x_{n}(j)\right)\right. \\
& \left.-\left(\sum_{j>j_{n}} M\left(\frac{k k_{n}}{k+k_{n}} x_{n}(j)\right)+\frac{\delta \varepsilon}{2(1+\bar{k})}\right)\right) \\
\geq & \frac{k+k_{n}}{k k_{n}}\left(\sum_{j>j_{n}} \frac{k}{k+k_{n}} M\left(k_{n} x_{n}(j)\right)\right. \\
& \left.-\sum_{j>j_{n}}(1-\delta) \frac{k}{k+k_{n}} M\left(k_{n} x_{n}(j)\right)-\frac{\delta \varepsilon}{2(1+\bar{k})}\right) \\
= & \frac{k+k_{n}}{k k_{n}}\left(\delta \sum_{j>j_{n}} \frac{k}{k+k_{n}} M\left(k_{n} x_{n}(j)\right)-\frac{\delta \varepsilon}{2(1+\bar{k})}\right) \\
\geq & \frac{2}{\bar{k}}\left(\frac{\delta \varepsilon}{1+\bar{k}}-\frac{\delta \varepsilon}{2(1+\bar{k})}\right)=\frac{\delta \varepsilon}{\bar{k}(1+\bar{k})},
\end{aligned}
$$

which is a contradiction. So (1) is true.

Since $k_{n}>1$, we have

$$
\lim _{j_{0} \rightarrow \infty} \sup _{n} \sum_{j>j_{0}} M\left(x_{n}(j)\right)=0 .
$$

Again from $M \in \Delta_{2}$ and [3], Th. 1.23,

$$
\lim _{j_{0} \rightarrow \infty} \sup _{n}\left\|x_{n}-\left[x_{n}\right]_{j_{0}}\right\|_{M}^{0}=0 .
$$

For arbitrary $\varepsilon>0$, take $j_{0}$ such that

$$
\sup _{n}\left\|x_{n}-\left[x_{n}\right]_{j_{0}}\right\|_{M}^{0}<\varepsilon / 3 .
$$

Since $\left\{\left[x_{n}\right]_{j_{0}}\right\}_{n=1}^{\infty}$ is a bounded set in a $j_{0}$-dimensional space, there exist $\left\{\bar{x}_{i}\right\}_{i=1}^{i_{0}} \subset\left\{x_{n}\right\}$ such that for any $n$, there is $i, 1 \leq i \leq i_{0}$, satisfying $\left\|\left[x_{n}\right]_{j_{0}}-\left[\bar{x}_{i}\right]_{j_{0}}\right\|_{M}^{0}<\varepsilon / 3$. Hence

$$
\left\|x_{n}-\bar{x}_{i}\right\|_{M}^{0} \leq\left\|\left[x_{n}\right]_{j_{0}}-\left[\bar{x}_{i}\right]_{j_{0}}\right\|_{M}^{0}+\left\|x_{n}-\left[x_{n}\right]_{j_{0}}\right\|_{M}^{0}+\left\|\bar{x}_{i}-\left[\bar{x}_{i}\right]_{j_{0}}\right\|_{M}^{0}<\varepsilon,
$$

that is, $\left\{\bar{x}_{i}\right\}_{i=1}^{i_{0}}$ is an $\varepsilon$-net of $\left\{x_{n}\right\}$. Thus $\left\{x_{n}\right\}$ is a compact set.

For Orlicz function spaces, criteria for CLUR points are more complicated.

Theorem 2. Let $x \in S\left(L_{M}^{0}\right)$. Then $x$ is a CLUR point iff

(a) $M \in \Delta_{2}$;

(b) $M \in \nabla_{2}$;

(c) $\mu\{t \in T: k|x(t)| \in(a, b)\}=0$, where $k \in\left[k_{x}^{*}, k_{x}^{* *}\right]$ and $[a, b]$ is an arbitrary $S A I$ of $M$; 
(d) $\mu\left\{t \in T: k|x(t)|=a^{\prime}\right.$ or $\left.k|x(t)|=b^{\prime}\right\}=0$, where $a^{\prime}$ (resp. $\left.b^{\prime}\right)$ is the left (resp. right) endpoint of an arbitrary SAI of $M$ satisfying $p_{-}\left(a^{\prime}\right)=p\left(a^{\prime}\right)$ $\left(\right.$ resp. $\left.p_{-}\left(b^{\prime}\right)=p\left(b^{\prime}\right)\right)$;

(e) If $\mu\{t \in T: k|x(t)|=a\}>0$, where $a$ is the left endpoint of some $S A I$ of $M$ satisfying $p_{-}(a)<p(a)$, then $\varrho_{N}\left(p_{-}(k|x|)\right)=1$;

(f) If $\mu\{t \in T: k|x(t)|=b\}>0$, where $b$ is the right endpoint of some $S A I$ of $M$ satisfying $p_{-}(b)<p(b)$, then $\varrho_{N}(p(k|x|))=1$.

P r o of. Without loss of generality, assume $x(t) \geq 0(t \in T)$.

Necessity. The proof of (a), (b) is similar to (a), (b) of Theorem 1.

If (c) is not true then there is an SAI $[a, b]$ of $M$ such that $\mu\{t \in T$ : $k x(t) \in(a, b)\}>0$. Take $\varepsilon>0$ so small that the set $E=\{t \in T$ : $k x(t) \in[a+\varepsilon, b-\varepsilon]\}$ has positive measure. Divide $E$ into two disjoint measurable subsets $E_{1}^{1}$ and $E_{2}^{1}$ such that $\mu E_{1}^{1}=\mu E_{2}^{1}$; divide $E_{1}^{1}$ and $E_{2}^{1}$ into disjoint measurable subsets $E_{1}^{2}, E_{2}^{2}$ and $E_{3}^{2}, E_{4}^{2}$ respectively such that $\mu E_{1}^{2}=\mu E_{2}^{2}$ and $\mu E_{3}^{2}=\mu E_{4}^{2}, \ldots$; divide $E_{i}^{n-1}$ into two disjoint measurable subsets $E_{2 i-1}^{n}, E_{2 i}^{n}$ such that $\mu E_{2 i-1}^{n}=\mu E_{2 i}^{n}\left(i=1, \ldots, 2^{n-1}\right)$. Let

$$
\begin{aligned}
x_{n}= & \left.x\right|_{T \backslash E}+\left.(x+\varepsilon /(2 k))\right|_{\bigcup_{i=1}^{2 n-1} E_{2 i-1}^{n}} \\
& +\left.(x-\varepsilon /(2 k))\right|_{\bigcup_{i=1}^{2 n-1} E_{2 i}^{n}} .
\end{aligned}
$$

It is easy to see $\varrho_{N}\left(p_{-}\left(k x_{n}\right)\right)=\varrho_{N}\left(p_{-}(k x)\right)$ and $\varrho_{N}\left(p\left(k x_{n}\right)\right)=\varrho_{N}(p(k x))$. Hence $k \in\left[k_{x_{n}}^{*}, k_{x_{n}}^{* *}\right](n=1,2, \ldots)$. Therefore

$$
\begin{aligned}
\left\|x_{n}\right\|_{M}^{0}= & \frac{1}{k}\left(1+\varrho_{M}\left(k x_{n}\right)\right) \\
= & \frac{1}{k}\left(1+\varrho_{M}\left(\left.k x\right|_{T \backslash E}\right)+\int_{\bigcup_{i=1}^{2 n-1} E_{2 i-1}^{n}} M(k x(t)+\varepsilon / 2) d \mu\right. \\
& \left.+\int_{\bigcup_{i=1}^{2 n-1} E_{2 i}^{n}} M(k x(t)+\varepsilon / 2) d \mu\right) \\
= & \frac{1}{k}\left(1+\varrho_{M}(k x)+p(a) \frac{\varepsilon}{2} \cdot \frac{\mu E}{2}-p(a) \frac{\varepsilon}{2} \cdot \frac{\mu E}{2}\right)=\|x\|_{M}^{0}=1 .
\end{aligned}
$$

Obviously $k \in\left[k_{\left(x+x_{n}\right) / 2}^{*}, k_{\left(x+x_{n}\right) / 2}^{* *}\right](n=1,2, \ldots)$. We have

$$
\left\|\frac{x+x_{n}}{2}\right\|_{M}^{0}=\frac{1}{k}\left(1+\varrho_{M}\left(k \frac{x+x_{n}}{2}\right)\right)=\|x\|_{M}^{0}=1 .
$$

That is, $\left\|x+x_{n}\right\|_{M}^{0}=2$. But for $m \neq n$,

$$
\left\|x_{n}-x_{m}\right\|_{M}^{0}=\frac{\varepsilon}{2 k} \cdot \frac{\mu E}{2} N^{-1}\left(\frac{2}{\mu E}\right)
$$

(see [1], Ex. 1.22). Thus $\left\{x_{n}\right\}$ is not a compact set. 
If (d) is not true then there is a SAI $\left[a^{\prime}, b^{\prime}\right]$ of $M$ with $p_{-}\left(a^{\prime}\right)=p\left(a^{\prime}\right)$ and $E=\left\{t \in T: k x(t)=a^{\prime}\right\}$ of positive measure. Take $c^{\prime}$ with $a^{\prime}<c^{\prime}<b^{\prime}$. Divide $E$ as above and put

$$
x_{n}=\left.x\right|_{T \backslash E}+\left.\frac{a^{\prime}}{k}\right|_{\bigcup_{i=1}^{2 n-1} E_{2 i-1}^{n}}+\left.\frac{c^{\prime}}{k}\right|_{\bigcup_{i=1}^{2 n-1} E_{2 i}^{n}} \quad(n=1,2, \ldots) .
$$

We have $\varrho_{N}\left(p_{-}\left(k x_{n}\right)\right)=\varrho_{N}\left(p_{-}(k x)\right)$ and $\varrho_{N}\left(p\left(k x_{n}\right)\right)=\varrho_{N}(p(k x))$. So $k \in$ $\left[k_{x_{n}}^{*}, k_{x_{n}}^{* *}\right](n=1,2, \ldots)$. Since $M \in \Delta_{2}$ there is $y \in S\left(I_{N}\right)$ such that $\langle x, y\rangle=$ $\|x\|_{M}^{0}=1$. From [1], Th. 1.80, we have $\varrho_{N}(y)=1$ and $p_{-}(k x(t)) \leq y(t) \leq$ $p(k x(t))$ (a.e. $t \in T)$. Now $p_{-}\left(k x_{n}(t)\right) \leq y(t) \leq p\left(k x_{n}(t)\right)(n=1,2, \ldots)$ too. Again by [1], Th. 1.80, $y$ is a supporting functional of $x_{n}(n=1,2, \ldots)$. Thus

$$
2 \geq\left\|x+\frac{x_{n}}{\left\|x_{n}\right\|_{M}^{0}}\right\|_{M}^{0} \geq \int_{T}\left(x(t)+\frac{x_{n}(t)}{\left\|x_{n}\right\|_{M}^{0}}\right) y(t) d \mu=2 \quad(n=1,2, \ldots),
$$

i.e. $\left\|x+x_{n} /\right\| x_{n}\left\|_{M}^{0}\right\|_{M}^{0}=2$. Clearly $\left\|x_{n}\right\|_{M}^{0}=A<\infty(n=1,2, \ldots)$ and for $m \neq n$,

$$
\left\|\frac{x_{n}}{\left\|x_{n}\right\|_{M}^{0}}-\frac{x_{m}}{\left\|x_{m}\right\|_{M}^{0}}\right\|_{M}^{0}=\frac{c^{\prime}-a^{\prime}}{A} \cdot \frac{\mu E}{2} N^{-1}\left(\frac{2}{\mu E}\right) .
$$

Hence $\left\{x_{n} /\left\|x_{n}\right\|_{M}^{0}\right\}$ is not a compact set.

Similarly we can prove that $\mu\left\{t \in T: k x(t)=b^{\prime}\right\}=0$.

If (e) is not true then there is a SAI $[a, b]$ of $M$ with $p_{-}(a)<p(a)$, $E_{0}=\{t \in T: k x(t)=a\}$ of positive measure and $\varrho_{N}\left(p_{-}(k x)\right)<1$ (notice that we always have $\varrho_{N}\left(p_{-}(k x)\right) \leq 1$ by the definition of $k$ ). Take $E \subset E_{0}$ with $\mu E>0$ such that

$$
\begin{aligned}
\int_{T \backslash E} N\left(p_{-}(k x(t))\right) d \mu+\int_{E} N & p(k x(t))) d \mu \\
& =\int_{T \backslash E} N\left(p_{-}(k x(t))\right) d \mu+N(p(a)) \mu E \leq 1 .
\end{aligned}
$$

Divide $E$ as above. Take $c$ with $a<c<b$ and put

$$
x_{n}=\left.x\right|_{T \backslash E}+\left.\frac{a}{k}\right|_{\bigcup_{i=1}^{2 n-1} E_{2 i-1}^{n}}+\left.\frac{c}{k}\right|_{\bigcup_{i=1}^{2 n-1} E_{2 i}^{n}} \quad(n=1,2, \ldots) .
$$

Then

$$
\varrho_{N}\left(p_{-}\left(k x_{n}\right)\right)=\int_{T \backslash E} N\left(p_{-}(k x(t))\right) d \mu+N(p(a)) \mu E \leq 1
$$

and

$$
\varrho_{N}\left(p\left(k x_{n}\right)\right)=\varrho_{N}(p(k x)) \geq 1 .
$$


So $k \in\left[k_{x_{n}}^{*}, k_{x_{n}}^{* *}\right](n=1,2, \ldots)$. Since

$$
\begin{gathered}
\int_{T \backslash E} N\left(p_{-}(k x(t))\right) d \mu+N(p(a)) \mu E \leq 1, \\
\int_{T \backslash E} N(p(k x(t))) d \mu+N(p(a)) \mu E=\varrho_{N}(p(k x(t))) \geq 1,
\end{gathered}
$$

there is $y \in L_{N}$ such that

$$
y(t)=p(a) \quad(t \in E), \quad p_{-}(k x(t)) \leq y(t) \leq p(k x(t)) \quad(t \in T \backslash E)
$$

and $\varrho_{N}(y)=1$. By [1], Th. 1.80, $y$ is a supporting functional of $x$. Obviously $p_{-}\left(k x_{n}(t)\right) \leq y(t) \leq p\left(k x_{n}(t)\right)$ (a.e. $\left.t \in T, n=1,2, \ldots\right)$, so $y$ is a supporting functional of $x_{n}(n=1,2, \ldots)$ too. Thus

$$
\left\|x+\frac{x_{n}}{\left\|x_{n}\right\|_{M}^{0}}\right\|_{M}^{0} \geq \int_{T}\left(x(t)+\frac{x_{n}(t)}{\left\|x_{n}\right\|_{M}^{0}}\right) y(t) d \mu=2 \quad(n=1,2, \ldots) .
$$

Assume $\left\|x_{n}\right\|_{M}^{0}=A(n=1,2, \ldots)$. Then for $m \neq n$,

$$
\left\|\frac{x_{n}}{\left\|x_{n}\right\|_{M}^{0}}-\frac{x_{m}}{\left\|x_{m}\right\|_{M}^{0}}\right\|_{M}^{0}=\frac{c-a}{A} \cdot \frac{\mu E}{2} N^{-1}\left(\frac{2}{\mu E}\right),
$$

which means $\left\{x_{n} /\left\|x_{n}\right\|_{M}^{0}\right\}$ is not a compact set.

The proof of (f) is similar to (e).

Sufficiency. By [6], $x$ is a LUR point. Then of course it is a CLUR point.

Finally, we discuss a criterion for CLUR points in Orlicz function spaces with Luxemburg norm.

Theorem 3. Let $x \in S\left(L_{M}\right)$. Then $x$ is a CLUR point iff

(a) $M \in \Delta_{2}$;

(b) $\mu\{t \in T:|x(t)| \in(a, b)\}=0$, where $[a, b]$ is an arbitrary SAI of $M$;

(c) $\mu\{t \in T:|x(t)|=b\}=0$ for the right endpoint $b$ of any SAI of $M$, or $\mu\{t \in T:|x(t)|=a\}=0$ for the left endpoint $a$ of any SAI of $M$, and $M \in \nabla_{2}$.

Proof. Still assume $x(t) \geq 0(t \in T)$.

Sufficiency. By [5], $x$ is a LUR point. Then certainly it is a CLUR point.

Necessity. The proofs of (a) and (b) are similar to (a) and (c) in Theorem 2 , respectively.

If (c) is not true, we consider the following two cases:

I. Both $E=\{t \in T: x(t)=b\}$ and $F=\{t \in T: x(t)=c\}$ have positive measure, where $(a, b)$ and $(c, d)$ are two SAI of $M$. Take $s \in(a, b)$ and $r \in(c, d)$ satisfying $(M(b)-M(s)) \mu E=(M(r)-M(c)) \mu F$. Divide $E$ 
and $F$ as above and put

We have

$$
\begin{aligned}
x_{n}= & \left.x\right|_{T \backslash(E \cup F)}+\left.b\right|_{\bigcup_{i=1}^{2 n-1} E_{2 i-1}^{n}}+\left.s\right|_{\bigcup_{i=1}^{2 n-1} E_{2 i}^{n}} \\
& +\left.r\right|_{\bigcup_{i=1}^{n-1} F_{2 i-1}^{n}}+\left.c\right|_{\bigcup_{i=1}^{2 n-1} F_{2 i}^{n}} \quad(n=1,2, \ldots) .
\end{aligned}
$$

$$
\begin{aligned}
\varrho_{M}\left(x_{n}\right) & =\varrho_{M}\left(\left.x\right|_{T \backslash(E \cup F)}\right)+(M(b)+M(s)) \frac{\mu E}{2}+(M(r)+M(c)) \frac{\mu F}{2} \\
& =\varrho_{M}\left(\left.x\right|_{T \backslash(E \cup F)}\right)+M(b) \mu E+M(c) \mu F=\varrho_{M}(x)=1 .
\end{aligned}
$$

So $\left\|x_{n}\right\|_{M}=1(n=1,2, \ldots)$. Moreover

$$
\begin{aligned}
\varrho_{M}\left(\frac{x_{n}+x}{2}\right) & =\varrho_{M}\left(\left.x\right|_{T \backslash(E \cup F)}\right)+M\left(\frac{b+s}{2}\right) \mu E+M\left(\frac{r+c}{2}\right) \mu F \\
& =\varrho_{M}\left(\left.x\right|_{T \backslash(E \cup F)}\right)+\frac{M(b)+M(s)}{2} \mu E+\frac{M(r)+M(c)}{2} \mu F=1 .
\end{aligned}
$$

So $\left\|x+x_{n}\right\|_{M}=2(n=1,2, \ldots)$. But for $m \neq n$,

$$
\varrho_{M}\left(x_{n}-x_{m}\right)=M(c-a) \frac{\mu E}{2}+M(b-d) \frac{\mu F}{2} .
$$

Therefore $\left\{x_{n}\right\}$ is not a compact set.

II. $E=\{t \in T: x(t)=b\}$ has positive measure, where $[a, b]$ is a SAI of $M$ and $M \notin \nabla_{2}$.

By [1], Th. 1.13, there exist $u_{n} \rightarrow \infty$ such that

$$
M\left(\frac{u_{n}}{2}\right)>\left(1-\frac{1}{n}\right) \frac{M(u)}{2} \quad(n=1,2, \ldots) .
$$

Since $\varrho_{M}\left(\left.x\right|_{T \backslash E}\right)+M(b) \mu E=\varrho_{M}(x)=1$, we can take $E_{n} \subset E$ such that

$$
\varrho_{M}\left(\left.x\right|_{T \backslash E}\right)+M\left(u_{n}-b\right) \mu E_{n}+M(a) \mu\left(E \backslash E_{n}\right)=1 \quad(n=1,2, \ldots) .
$$

It is easy to see that $\mu E_{n} \rightarrow 0(n \rightarrow \infty)$. Without loss of generality we can assume that $E_{n} \cap E_{m}=\emptyset(m \neq n)$. Put

$$
x_{n}=\left.x\right|_{T \backslash E}+\left.a\right|_{E \backslash E_{n}}+\left.\left(u_{n}-b\right)\right|_{E_{n}} \quad(n=1,2, \ldots) .
$$

Then $\varrho_{M}\left(x_{n}\right)=1$ and

$$
\begin{aligned}
\varrho_{M}\left(\frac{x_{n}+x}{2}\right)= & \varrho_{M}\left(\left.x\right|_{T \backslash E}\right)+M\left(\frac{b+a}{2}\right) \mu\left(E \backslash E_{n}\right)+M\left(\frac{u_{n}}{2}\right) \mu E_{n} \\
\geq & \varrho_{M}\left(\left.x\right|_{T \backslash E}\right)+\frac{M(b)+M(a)}{2} \mu\left(E \backslash E_{n}\right)+\left(1-\frac{1}{n}\right) \frac{M\left(u_{n}\right)}{2} \mu E_{n} \\
= & \frac{1}{2}\left[\varrho_{M}\left(\left.x\right|_{T \backslash E}\right)+M(b) \mu\left(E \backslash E_{n}\right)\right. \\
& \left.+\varrho_{M}\left(\left.x\right|_{T \backslash E}\right)+M(a) \mu\left(E \backslash E_{n}\right)+\left(1-\frac{1}{n}\right) M\left(u_{n}\right) \mu E_{n}\right] \\
\rightarrow & 1 \quad(n \rightarrow \infty) .
\end{aligned}
$$


Hence $\left\|x_{n}\right\|_{M}=1(n=1,2, \ldots),\left\|x_{n}+x\right\|_{M} \rightarrow 2(n \rightarrow \infty)$. But for any $n, m, m \neq n$,

$$
\begin{aligned}
\varrho_{M}\left(x_{n}-x_{m}\right) & \geq \varrho_{M}\left(\left.\left(x_{n}-x_{m}\right)\right|_{E_{n}}\right) \\
& =M\left(u_{n}-b-a\right) \mu E_{n}=M\left(u_{n}-b\right) \mu E_{n}-o(1 / n) \\
& =\left(1-\varrho_{M}\left(\left.x\right|_{T \backslash E}\right)\right)-M(a) \mu\left(E \backslash E_{n}\right)-o(1 / n) \\
& =M(b) \mu E-M(a) \mu\left(E \backslash E_{n}\right)-o(1 / n) \\
& \geq(M(b)-M(a)) \mu E-o(1 / n) \\
& \geq \frac{1}{2}(M(b)-M(a)) \mu E
\end{aligned}
$$

for $n$ large enough. This means $\left\{x_{n}\right\}$ is not a compact set.

Combining Theorems 1-3 of this paper and Theorem 6 of [2] we see that being a CLUR point is equivalent to being a LUR point in Orlicz function spaces, while in Orlicz sequence spaces a CLUR point does not even have to be an extreme point. Furthermore we can get

Corollary 1. $L_{M}^{0}$ (and $\left.L_{M}\right)$ is CLUR iff it is LUR.

Corollary 2. $I_{M}^{0}$ is CLUR iff it is reflexive.

\section{References}

[1] S. T. Chen, Geometry of Orlicz spaces, Dissertationes Math. 356 (1996).

[2] Y. A. Cui, H. Hudzik and C. Meng, On some local geometry of Orlicz sequence spaces equipped with the Luxemburg norm, Acta Math. Hungar. 80 (1998), 143-154.

[3] J. Y. Fu and W. Y. Zhang, On weakly compact locally uniformly rotund spaces, J. Math. (Wuhan) 6 (1986), 285-290; MR 88b:46028.

[4] B. B. Panda and O. P. Kapoor, A generalization of local uniform convexity of the norm, J. Math. Anal. Appl. 52 (1975), 300-308.

[5] T. F. Wang, Z. D. Ren and Y. L. Zhang, On UR points and WUR points of Orlicz spaces, J. Math. (Wuhan) 13 (1993), 443-452; MR 95h:46049.

[6] T. F. Wang, Z. R. Shi and Q. D. Wang, On $W^{*} U R$ point and $U R$ point of Orlicz spaces with Orlicz norm, Collect. Math. 44 (1993), 279-299.

Department of Applied Mathematics Jilin University of Technology 130025 Changchun, P.R. China E-mail: zjwqd@public.cc.jl.cn
Harbin University of Sciences and Technology P.O. Box 123 150080 Harbin, P.R. China E-mail: wangtf@ems.hrbmu.edu.cn 